\title{
Houchang Hassan-Yari
}

$\mathrm{BA}, \mathrm{MA}, \mathrm{PhD}$,

Professeur et directeur, département de science politique et d'économique Collège militaire royal du Canada à Kingston

\section{(4-5 août 2006)}

\section{"Crise au Proche- Orient”}

Un document produit en version numérique par Jean-Marie Tremblay, bénévole, professeur de sociologie au Cégep de Chicoutimi

Courriel: jean-marie tremblay@uqac.ca

Site web pédagogique : http://www.uqac.ca/jmt-sociologue/

Dans le cadre de la collection: "Les classiques des sciences sociales"

Site web: http://classiques.uqac.ca/

Une collection développée en collaboration avec la Bibliothèque Paul-Émile-Boulet de l'Université du Québec à Chicoutimi

Site web: http://bibliotheque.uqac.ca/ 
Cette édition électronique a été réalisée par Jean-Marie Tremblay, bénévole, professeur de sociologie au Cégep de Chicoutimi à partir de l'article de :

Houchang Hassan-Yari

Professeur et directeur, département de science politique, Collège militaire royal du Canada à Kingston

“Crise au Proche-Orient”. Le Devoir, Montréal, Édition du vendredi, 4 août 2006, page A9 -idées, et du samedi le 5 août 2006 en page B5 - idées.

[Autorisation formelle accordée par l'auteur de diffuser, dans Les Classiques des sciences sociales, le texte de ces deux articles le 4 août 2006.]

A. Courriel : hassan-yari-h@rmc.ca

Polices de caractères utilisée :

Pour le texte: Times New Roman, 14 points.

Pour les citations : Times New Roman 12 points.

Pour les notes de bas de page : Times New Roman, 12 points.

Édition électronique réalisée avec le traitement de textes Microsoft Word 2004 pour Macintosh.

Mise en page sur papier format : LETTRE (US letter), 8.5'’ x 11'’)

Édition numérique réalisée le 5 août 2006 à Chicoutimi, Ville de Saguenay, province de Québec, Canada.

\section{f. Fait avec}




\section{LE DEV@⿺}

\section{“Crise au Proche-Orient”}

Houchang Hassan-Yari

Professeur et directeur, département de science politique,

Collège militaire royal du Canada à Kingston

\section{(1) Pour qui le Liban brûle-t-il ?}

La crise actuelle dans les territoires palestiniens occupés, au Liban et en Israël, prouve la futilité des stratégies militaires jusqu'au-boutistes et des demi-solutions imposées

Le Devoir, Montréal, Édition du vendredi, 4 août 2006, page A9.

http://www.ledevoir.com/cgi-bin/imprimer?path=/2006/08/04/115134.html

Houchang Hassan-Yari

Professeur et directeur, département de science politique et d'économique, Collège militaire royal du Canada à Kingston.

Article diffusé avec l'autorisation de l'auteur accordée le 4 août 2006.

Courriel : hassan-yari-h@rmc.ca

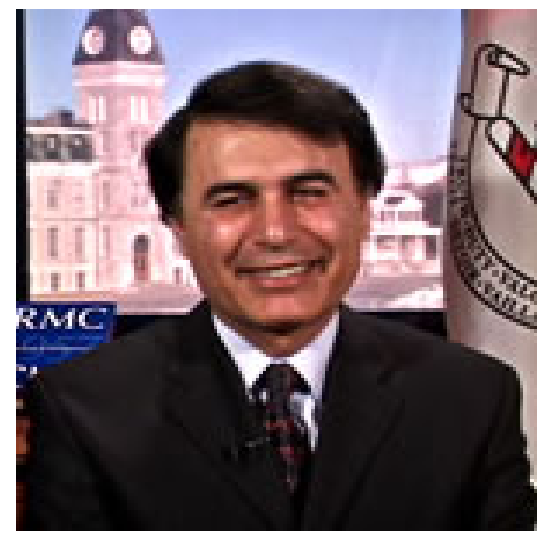

Premier de deux textes. Maillon faible du conflit israélo-arabe, le Liban paie cher son impuissance militaire. Pris entre trois feux, Israël, la Syrie et l'inspiration nationale légitime palestinienne, le pays du Cèdre a subi plusieurs invasions et interventions étrangères. Les intervenants ont toujours recouru à la religion pour justifier leurs méfaits. 
La France, puissance mandataire et héritière de l'Empire ottoman dans cette partie du Levant, a taillé une constitution qui a gravé le confessionnalisme sur le corps de la structure du pouvoir au Liban. Les interventions américaines en 1958 et en 1983 étaient surtout destinées à creuser davantage le fossé confessionnel en appuyant le pouvoir pro-occidental chrétien. Les deux mésaventures malheureuses n'ont renforcé ni la position des protégés américains, ni celle des États-Unis au Liban et au Moyen-Orient.

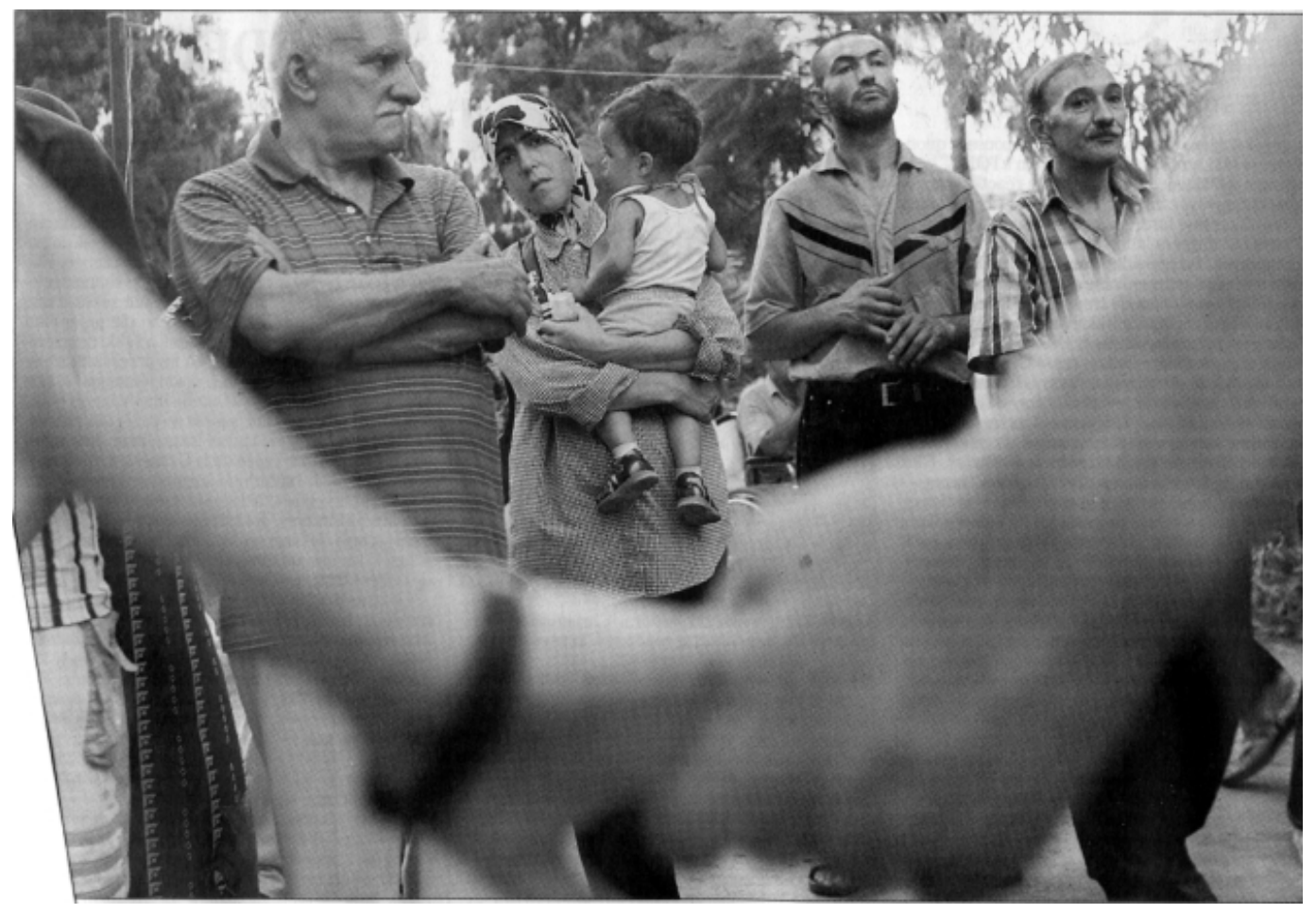

Des réfugiés du Sud-Liban attendent dans un parc de Beyrouth la distribution de couvertures pour leur permettre de passer la nuit au chaud. (Shannon Stapleton, euters).

Les plus importantes invasions du Liban par Israël, en 1978, en 1982 et en 1996, ont donné naissance à la création du Hezbollah et ont produit les massacres de la population palestinienne dans les camps de réfugiés de Sabra et Shatila (1982) et des Libanais à Cana (1996). L'aventure de 1982 a duré 88 jours et a tué 18000 personnes. Toutes ces invasions, qui avaient pour objectif premier la destruction de l'OLP et du Hezbollah, ont produit des résultats opposés. L'organisation palestinienne devient partenaire de paix pour l'État hébreux et la 
volonté palestinienne de se libérer du joug de l'occupant se poursuit dans les territoires occupés. Le Hezbollah, pour sa part, émerge comme la seule force arabe contraignant Israël à évacuer un territoire arabe occupé.

Les trois invasions ont été sanctionnées par Washington, qui se réservait le droit d'imposer un règlement aux belligérants, tout en s'affairant à maintenir la suprématie militaire stratégique d'Israël sur les Arabes. L'intervention américaine arrivait à un moment critique, alors que survenaient des bavures israéliennes où la condition humaine des gens envahis soulevait l'indignation de l'opinion publique internationale.

Sur le plan stratégique, les invasions -- qui n'ont ni altéré sérieusement les rapports de force ni créé de nouvelles réalités durables sur le terrain favorisant la position des groupes libanais pro-israéliens -compliquent tout règlement pacifique permanent des conflits sur la base d'une légalité internationale.

La crise actuelle dans les territoires palestiniens occupés, au Liban et en Israël, prouve la futilité des stratégies militaires jusqu'auboutistes et des demi-solutions imposées.

La résolution de ces conflits serait plus facile que ce qu'on laisse penser, coûterait moins cher et produirait de meilleurs résultats si un " grand jeu » n'opposait pas les aspirations régionales aux attentes des grandes puissances.

\section{Jeu des enjeux}

Région sensible et stratégique, le Moyen-Orient était un enjeu important durant les décennies de la guerre froide. Israël constituait un élément central d'une alliance très hétéroclite proaméricaine qui englobait des pays arabes comme l'Égypte et l'Arabie Saoudite tandis que le Front de refus, la Syrie, l'OLP et d'autres, s'alignait sur l'Union soviétique. La fin du système bipolaire coïncide avec la présence écrasante des États-Unis dans tous les aspects de la vie régionale. 
Les erreurs stratégiques du régime irakien en envahissant l'Iran (1980-1988), puis le Koweït (le 2 août 1990) et son programme de fabrication des armes de destruction massive, que les États-Unis utilisent comme prétexte pour remodeler tout le Moyen-Orient sur le schéma des conservateurs extrémistes au pouvoir à Washington, accélèrent la descente aux enfers des peuples de cette région et consolident la domination mondiale américaine.

Le projet de la démocratisation du grand Moyen-Orient doit répondre aux exigences politico-militaro-économiques des États-Unis. Dans cette nouvelle configuration qui devrait conduire à une Pax Americana, toute voix dissidente doit être étouffée et la soumission de la région sera absolue.

Un des objectifs de la présente guerre consiste à éliminer une de ces voix indésirables, celle du Hezbollah. Si Israël réussit à écraser la capacité militaire du mouvement et à le neutraliser dans l'équation politique au Liban avec la complaisance américaine et de la majorité des États arabes, le gouvernement de Tel-Aviv marquerait des points importants. Le réaménagement de la partition du pouvoir au Liban défavoriserait, comme dans le passé, les chiites et pourrait faciliter les tentatives des éléments libanais favorables à un règlement de conflit avec Israël. Cette stratégie, résultante de l'invasion de 1982, a échoué dans le sang.

La deuxième conséquence d'une victoire décisive éventuelle d'Israël serait l'isolement de la Syrie et la perte de ses droits légitimes sur son territoire occupé, les hauteurs du Golan.

Le troisième résultat de ce développement serait une politique militariste plus agressive dans les territoires palestiniens occupés. Le premier ministre d'Israël, Éhoud Olmert, serait conforté dans sa visée unilatéraliste d'imposer la carte territoriale et politique d'un futur État en Palestine. Les faits historiques nous enseignent l'inefficacité à moyen et à long terme d'une telle politique. En d'autres mots, la question palestinienne demeurera le noeud gordien de la situation bloquée au Moyen-Orient. 
Le renforcement des régimes régressifs et proaméricains arabes sera une conséquence de ce développement. Cela ne fera que retarder momentanément l'inévitable changement politique et économique dans ces pays. Une radicalisation des mouvements contestataires, de couleur religieuse ou nationaliste, restera la seule issue de la situation bloquée.

Une relecture de l'histoire des pays du Moyen-Orient depuis les années 60 montre le recul de la modération au fil des échecs et défaites arabes envers Israël et les États-Unis. Donc, la vie d'une victoire israélienne, qui est en fait celle des États-Unis dans l'analyse des peuples de la région, ne sera que de courte durée. La prochaine tempête sera plus dévastatrice; elle ne laissera pas grand espace à la raison et à la modération.

\section{La Syrie d'abord}

Indépendamment de la couleur politique des dirigeants de ce pays, y compris dans la faible probabilité d'un changement de régime à Damas, la libération du Golan restera l'objectif suprême de la politique étrangère et de la défense de la Syrie. La victoire politique déjà acquise du Hezbollah dans l'opinion publique arabo-musulmane est une bonne nouvelle pour Damas.

L'éradication très peu probable du Hezbollah serait un coup très dur pour la Syrie. Contrairement aux déclarations israélo-américaines sur la subordination du Hezbollah au diktat de Damas, la Syrie reste un joueur important dans la région et le renforcement de son isolement ne sert ni les intérêts combinés israélo-américains ni ceux des régimes arabes dits pro-occidentaux.

\section{Et l'Iran}

Le régime islamique a des rapports idéologiques et organiques avec le Hezbollah, en plus des relations politiques et stratégiques que celui-ci entretient avec la Syrie. La Constitution iranienne a fait, pour le gouvernement de Téhéran, une obligation de solidarité avec les déshérités du monde, notamment les musulmans. 
Cela n'a cependant pas empêché la République islamique d'être très pragmatique dans sa politique étrangère, de l'acceptation de la résolution 598 du Conseil de sécurité des Nations unies, qui mettait fin aux hostilités avec l'Irak, au présent conflit israélo-libanais, où le régime de Téhéran a clairement déclaré son refus d'aider le Hezbollah militairement et a empêché le départ des étudiants iraniens volontaires prêts à se martyriser dans la guerre contre «l'arrogance [États-Unis] et le régime sioniste[Israël]».

Même si le déclenchement des hostilités par le Hezbollah était destiné à faire oublier la question nucléaire iranienne (une analyse israélo-américaine que nous ne partageons pas), il reste que le résultat de la crise actuelle aura un impact direct sur les rapports de l'Iran avec le reste du monde. Un Hezbollah sorti vivant de l'épreuve actuelle sera une carte gagnante avec une portée très limitée pour l'Iran dans ses rapports extérieurs.

\section{Que faire?}

Si le sang versé des innocents libanais et israéliens conduit à une solution durable et juste du conflit israélo-arabe, la peine des enfants d'Abraham serait moindre. Si l'occupation des territoires arabes continuait et que l'insécurité d'Israël devait se traduire par d'autres destructions, le monde restera confronté à d'autres tragédies comme Cana.

La solution à ce conflit existe déjà dans le livre des résolutions de l'ONU : Israël libère les territoires arabes occupés en 1967, les Arabes reconnaissent l'existence d'Israël. Ce dernier ne doit pas choisir quelle résolution doit être respectée ; il faut appliquer toutes les résolutions ou vivre dans la peur.

\section{Demain : La politique canadienne.}




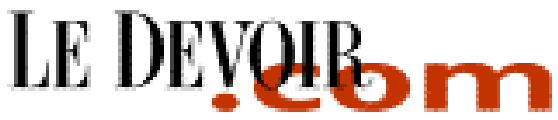

\section{“Crise au Proche-Orient”}

Houchang Hassan-Yari

Professeur et directeur, département de science politique,

Collège militaire royal du Canada à Kingston

\section{(2) Le Canada et la guerre Israël Liban: rupture ou continuité?}

Édition du samedi 5 et du dimanche 6 août 2006, page B5.

http://www.ledevoir.com/2006/08/05/115240.html

\section{Dernier de deux textes.}

Houchang Hassan-Yari

Professeur et directeur, département de science politique et d'économique, Collège militaire royal du Canada à Kingston.

Article diffusé avec l'autorisation de l'auteur accordée le 4 août 2006.

\section{Courriel : hassan-yari-h@rmc.ca}

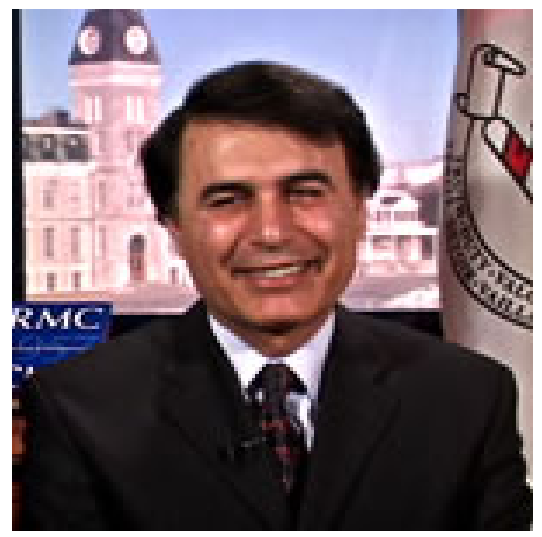

Ce texte cherche à comprendre la prise de position de l'actuel gouvernement canadien dans cette guerre en la comparant à la politique traditionnelle du pays. Il ne se veut donc pas un plaidoyer en faveur des deux parties dans un conflit dont les actes sont condamnables. Dans le présent climat surchauffé, cette précision me semble nécessaire. 


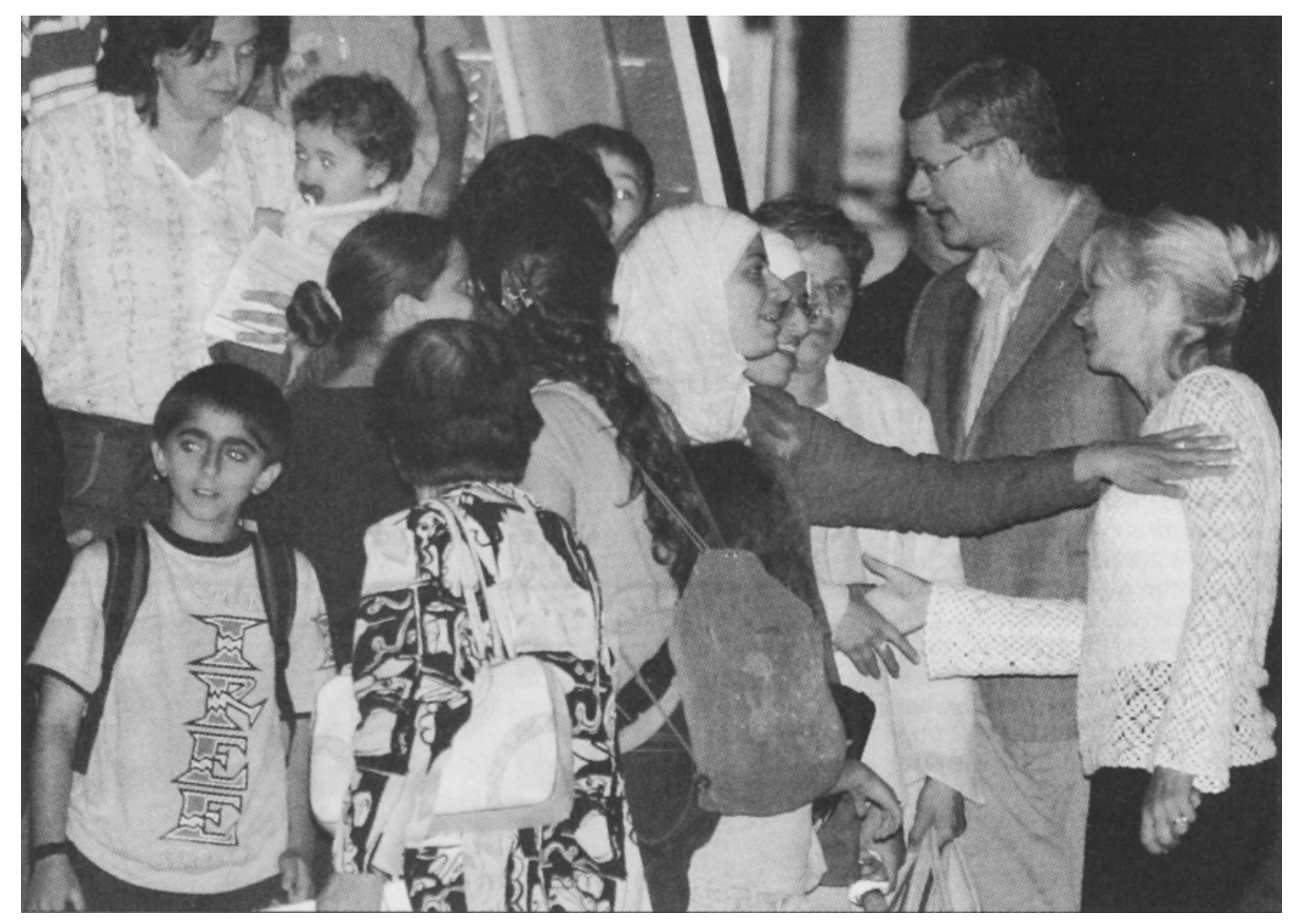

Stephen Harper en compagnie d'écacués libanais lors de leur retour au Canada, le 21 juillet. Le premier ministre avait autorisé l'utilisation d'un avion militaire canadien pour permettre l'évacuation de Canadiens d'origine libanaise. [Reuters]

La réflexion à laquelle donne lieu la politique du Canada est suscitée par la déclaration du premier ministre Harper, qui a qualifié de « mesurée » la réaction d'Israël à l'enlèvement de deux de ses soldats par le Hezbollah. L'acte du Hezbollah est condamnable quelles que soient les raisons invoquées par ce groupe, soit faire libérer quelques centaines de Libanais emprisonnés en Israël et mettre fin à l'occupation des fermes Shebaa par l'État hébreu.

\section{Faits et méfaits}

Quel que soit le démenti sans équivoque que les réalités sur le terrain libanais apportent à la déclaration du premier ministre, les propos du ministre des Affaires étrangères Peter MacKay sont encore plus explicites et vont plus loin. Pour lui, dans la crise actuelle, le choix est clair entre un gouvernement démocratiquement élu attaqué par des terroristes et un groupe de tueurs sans pitié. 
Examinons d'abord la question du principe de proportionnalité de la campagne militaire israélienne. Le premier ministre du Liban, dont le caractère démocratique est reconnu par le ministre MacKay, a présenté, le 3 août 2006, le bilan suivant de l'action «mesurée» d'Israël dans son pays : 900 morts, 3000 blessés, dont un tiers d'enfants, sans oublier la destruction de l'infrastructure économique du Liban. Chacun a le droit de croire ses sources : l'ONU et le reste du monde, qui dénoncent les excès israéliens, ou les États-Unis de George W. Bush, l'Angleterre de Tony Blair, l'Israël d'Ehud Olmert, etc., qui ne représentent même pas l'ensemble de l'opinion publique de leur propre pays !

Au-delà des considérations politiques et conjoncturelles, il existe des principes fondamentaux gouvernant la vie internationale qu'on ne saurait sacrifier. Il y a un droit de la guerre à respecter et des vies à épargner à tout prix. Israël et le Hezbollah violent ces normes.

Le choix du ministre des Affaires étrangères est partiel, partial et très hâtif. Le tableau du Moyen-Orient se compose de plusieurs couches; il est complexe et ne peut se réduire à une lutte manichéenne entre le bien et le mal. Rompant avec la diplomatie canadienne, qui s'est traditionnellement montrée nuancée et imaginative, le ministre a repris mot pour mot la description que fait Israël du mouvement libanais : « Le Hezbollah est un cancer qui ronge le Liban en détruisant sa stabilité et sa démocratie. »

On peut, et surtout on doit, condamner la violence des États et des organisations qui opèrent en dehors du cadre étatique, mais dans le cas qui nous occupe, une évaluation plus précise de la place du Hezbollah dans la société libanaise s'impose. Ce groupe a fait élire des députés au parlement libanais à l'occasion d'élections démocratiques et compte des ministres au sein du gouvernement. Rappelons qu'un autre gouvernement démocratiquement élu, celui du Hamas dans les territoires palestiniens, fait l'objet d'attaques quotidiennes du Tsahal, et ce dans l'indifférence quasi générale des États démocratiques. On peut choisir pour qui voter, mais on ne peut rejeter le résultat des élections. 


\section{Politique du passé}

En réduisant l'actuelle guerre à un conflit entre Israël et le Hezbollah, le ministre MacKay voit dans le Liban une victime collatérale et confirme que la position de son gouvernement est "classique ». Si, par ce terme, il entend la politique passée du Canada, un survol rapide de celle-ci est nécessaire en vue de mieux apprécier la position actuelle.

Bien qu'il faille remonter relativement loin pour trouver une première présence canadienne dans la région du Proche-Orient et en Afrique du Nord, c'est la question juive, devenue le problème palestinien, qui a plongé le Canada dans la dynamique de la région.

Si l'on fait abstraction du rôle central qu'a joué le représentant canadien, Ivan Cleveland Rand, au sein de la Commission spéciale des Nations unies sur la Palestine, lors de la création de l'État hébreu en Palestine en 1948, la politique du Canada vis-à-vis du conflit israéloarabe a souvent visé un certain équilibre. S'intéressant peu à la politique extérieure, le premier ministre Mackenzie King et son secrétaire d'État aux Affaires extérieures, Louis Saint-Laurent, ont laissé le dossier de la Palestine entre les mains de Lester B. Pearson, soussecrétaire d'État, et du juge Rand. Très proche de la cause sioniste dans le projet de partage de la Palestine, Pearson a donné le feu vert à Rand, qui est ainsi devenu l'un des architectes de la création du nouvel État. La crise de Suez en 1956 a fait de Pearson un homme d'État exceptionnel, au point de se voir décerner le prix Nobel de la paix (1957), après avoir proposé de créer une force des Nations unies pour le maintien de la paix.

Le gouvernement de John Diefenbaker (1957-1963) annonce une pause dans les activités diplomatiques du Canada dans la région, quoique le premier ministre continue d'admirer Israël. Réélu cette fois comme premier ministre (1963-1968), Pearson reste fidèle à l'État d'Israël tout en se disant ouvert, insistant sur l'impartialité du Canada dans le conflit israélo-arabe. 
La guerre de juin 1967 remet en question la sécurité mondiale et réactive la politique du Canada, qui joue un rôle dans la rédaction de la résolution 242 du Conseil de sécurité. Celle-ci voulait établir un équilibre dans le règlement du conflit en proposant la formule «terre contre paix ». Dès 1967, la résolution 242 devient le fondement de la politique régionale du Canada. Pearson maintiendra cette position en dépit des gestes de l'opposition conservatrice de Diefenbaker en faveur de l'abandon de ce qu'il appelait la neutralité canadienne face aux Arabes déterminés à détruire la race juive.

\section{La realpolitik de Trudeau}

L'arrivée de Pierre Elliott Trudeau au pouvoir marque une étape importante dans la politique étrangère, qui doit désormais servir l'intérêt du Canada. La " dépearsonisation » de cette politique constitue une rupture nette avec la pratique du passé et le triomphe de la realpolitik.

S'intéressant plutôt aux rapports économiques avec les pays arabes, Trudeau donne l'appui du Canada aux résolutions 242 et 338. Tout en réitérant le droit d'Israël à vivre en paix, le Canada reconnaît « les droits et les aspirations légitimes des Palestiniens ». Pierre Trudeau ne plie pas devant les pressions qu'exerce le premier ministre israélien Menachem Begin, lors de sa visite officielle à Ottawa, sur la question de la reconnaissance de Jérusalem comme capitale d'Israël.

L'interrègne de Joe Clark donne lieu à la controverse entourant le projet de transfert de l'ambassade canadienne de Tel-Aviv à Jérusalem. Après avoir cédé devant les pressions du lobby pro-transfert, le premier ministre Clark revient sur sa décision, qui allait à l'encontre de la lettre et l'esprit des résolutions 242 et 338, pierres angulaires de la politique canadienne dans le conflit opposant Israël et les États arabes, et qui s'était attiré les foudres de pays comme les États-Unis, lesquels avaient déconseillé le déménagement.

Le retour de Trudeau au pouvoir annonce une plus grande objectivité dans la politique canadienne. L'invasion du Liban par Israël en 1982 provoque l'envoi de trois lettres par Trudeau à son homologue israélien, dans lesquelles il condamne Israël et souligne sa vive oppo- 
sition à cette intervention. Il appelle du même souffle Israël à la modération.

Les mandats du premier ministre Brian Mulroney marquent une distance par rapport à la politique du gouvernement précédent. La position pro-israélienne du premier ministre contraste avec celle du secrétaire d'État aux Affaires extérieures, Joe Clark. Si Mulroney parle de retenue dans l'usage de la force par l'armée israélienne contre les Palestiniens dans le contexte de l'Intifada en 1987, Clark dénonce la répression brutale des Palestiniens dans les territoires occupés. Fait étonnant, le Canada de Mulroney-Clark reconnaîtra même l'OLP.

De leur côté, les gouvernements de Jean Chrétien et Paul Martin mettent en oeuvre une politique moyen-orientale très timide, axée sur les relations économiques.

Le duo Harper-MacKay se montre plus cohérent dans sa position pro-israélienne que les gouvernements précédents. Le rapprochement avec Washington, après des années assez difficiles sous les libéraux, pourrait constituer un élément expliquant les propos de Harper, qui ne sont pas sans rappeler les positions de Mulroney, lui aussi très proche d'un président américain pro-israélien.

Ce dont le Proche-Orient a besoin ces jours-ci, c'est un cessez-lefeu immédiat pour mettre fin à la folle tuerie à laquelle nous assistons aujourd'hui. Le Canada doit l'âge d'or de sa diplomatie à l'imagination de ses dirigeants. Les dirigeants actuels du Canada sont-ils à l'hauteur des défis que le Proche-Orient pose à la communauté internationale ?

Fin du texte 